Supporting Information

\title{
Uniaxial Extension of Ultra-thin Freestanding Polymer Films
}

R. Konane Bay, Alfred J. Crosby

Affiliations: Polymer Science and Engineering, University of Massachusetts, Amherst, MA

01003, USA.

*Correspondence to: acrosby@umass.edu

\section{Annealing Conditions}

The $151.5 \mathrm{kDa}$ PS films and $100 \mathrm{~nm} 1007 \mathrm{kDa}$ PS films are vacuumed annealed at $170^{\circ} \mathrm{C}$ for 25 minutes and slow cooled under vacuum. The $30 \mathrm{~nm} 1007 \mathrm{kDa}$ PS are vacuumed annealed at $150^{\circ} \mathrm{C}$ for 24 hours and slow cooled under vacuum.

Table S1. Laser cutting parameters for freestanding polystyrene (PS) films

\begin{tabular}{|c|c|c|c|c|}
\hline $\begin{array}{c}\text { Molecular Weight } \\
(\mathbf{k D a})\end{array}$ & $\begin{array}{c}\text { Avg. } \\
\text { Thickness } \\
(\mathbf{n m})\end{array}$ & Power $(\%)$ & Speed (\%) & $\begin{array}{c}\text { Point per inch } \\
(\mathbf{P P I})\end{array}$ \\
\hline 151.5 & 102 & 5 & 10 & 800 \\
\hline 151.5 & 79 & 5 & 10 & 800 \\
\hline 151.5 & 56 & 5 & 10 & 800 \\
\hline 151.5 & 31 & 1 & 1 & 1000 \\
\hline 1007 & 106 & 7 & 10 & 800 \\
\hline 1007 & 31 & 5 & 1 & 1000 \\
\hline
\end{tabular}




\section{'Dog-bone' geometry}
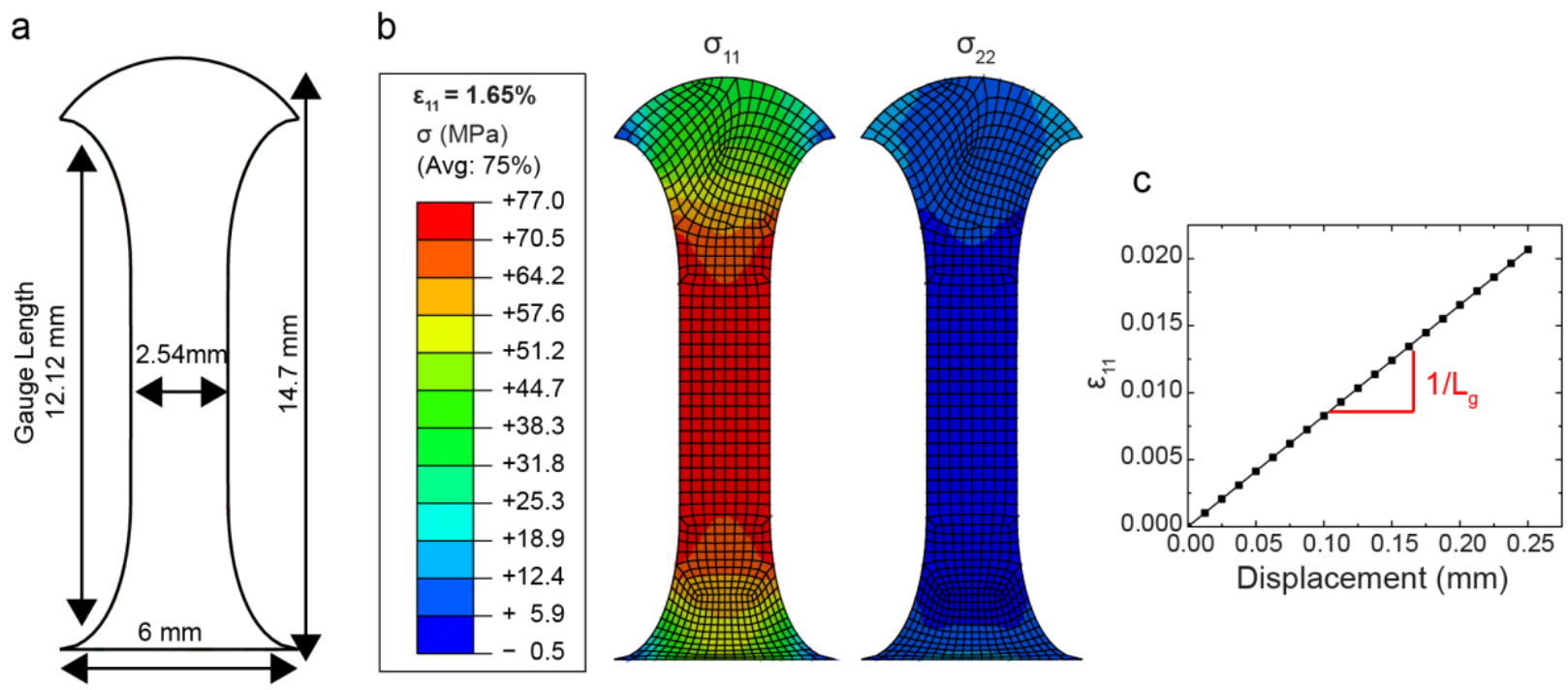

Figure S1. (a) Geometry of the freestanding PS film and (b) the PS FEA model. The stress at $1.65 \%$ strain is concentrated in the gauge regime. (c) Strain versus the applied displacement from the FEA where a linear fit is applied to find the slope, $1 / L_{\mathrm{g}}$ (Inverse of the gauge length). Using each elemental node in the gauge of FEA model, we calculated an average the gauge length of 12.12 mm. The width of the 'dog-bone' PS was measured to be $2.54 \mathrm{~mm}$.

The PS FEA models used to calculate the gauge length of the freestanding 'dog-bone' used twodimensional 4-node bilinear plane stress quadrilateral elements with reduced integration and hourglass control. We assign PS as linear elastic material with a Poisson's ratio of 0.33 and elastic modulus of $3.5 \mathrm{GPa}$. 


\section{Elastic Modulus $(E)$ Fitting}

In TUFF, there is occasional slack in the polymer film that can lead to a toe in the stress-strain curve. Although not all measured films have slack, to standardize the method that is used for measuring the modulus for all films, we implemented one approach. In Figure $3 a$ in the manuscript, $E$ is calculated from linear fits applied from 0.5 to $1.0 \%$ strain. In Table S2, we compare $E$ determined from the 0.5 to $1.0 \%$ strain to values obtained by linear fits from 0 to $1.0 \%$ strain in order to demonstrate how accounting for the slack in the linear fitting regime only slightly changes the elastic moduli values.

Table S2. Elastic Modulus (E) from different strain ranges

\begin{tabular}{|c|c|c|c|}
\cline { 3 - 4 } \multicolumn{2}{c|}{} & \multicolumn{2}{c|}{$E(\mathrm{GPa})$} \\
\hline $\begin{array}{c}\text { Molecular weight } \\
(\mathrm{kDa})\end{array}$ & $\begin{array}{c}\text { Thickness } \\
(\mathrm{nm})\end{array}$ & $\begin{array}{c}0.5 \% \text { to } \\
1.0 \% \text { strain }\end{array}$ & $\begin{array}{c}0 \% \text { to } \\
1.0 \% \\
\text { strain }\end{array}$ \\
\hline 151.5 & $102.2 \pm 1.3$ & $2.75 \pm 0.32$ & $2.69 \pm 0.35$ \\
\hline 151.5 & $79.0 \pm 0.8$ & $2.97 \pm 0.11$ & $2.93 \pm 0.39$ \\
\hline 151.5 & $56.0 \pm 2.0$ & $2.64 \pm 0.35$ & $2.50 \pm 0.35$ \\
\hline 151.5 & $30.8 \pm 1.6$ & $2.86 \pm 0.20$ & $2.68 \pm 0.28$ \\
\hline
\end{tabular}




\section{TUTTUT Experimental Method for the $30 \mathrm{~nm}$ thick films}

The $30 \mathrm{~nm}$ water supported PS films follow the same spin-coating and annealing protocol as the freestanding films. After annealing, the films are laser-cut on the mica substrate into a 'dogbone' shape. The films are floated off the mica substrate onto a water reservoir. The water level is lowered and the grip section of the 'dog-bone' is positioned into contact with a glass clamp coated with a PS film to promote adhesion between the 'dog-bone' film and the clamp. The glass clamp is rigidly attached to the reservoir. The 'dog-bone' is aligned with an extension piece attached to a cantilever and the reservoir is raised to bring the other grip section of the 'dogbone' into contact with the extension piece. The reservoir sits on a linear actuator that translates at a fixed speed and thereby a fixed strain rate $\left(\sim 5 \times 10^{-3} \mathrm{~s}^{-1}\right)$. As the film stretches, the deflection of the cantilever is measured and stress-strain is calculated from the film geometry (details are provided in reference 1). The length between the grip and clamp $\left(\mathrm{L}_{\mathrm{f}}\right)$ are measured and the gauge length $\left(\mathrm{Lg}_{\mathrm{g}}\right)$ is calculated from $\mathrm{Lg}_{\mathrm{g}}=\left(\mathrm{L}_{\mathrm{f}}-17.5\right)^{0.96}+16.4$ and the gauge width is 3.1 $\mathrm{mm}$. The thickness is measured with ellipsometry (index of refraction, $\mathrm{n}=1.59$ ). 


\section{Craze Stability Calculation}

We calculate the craze stability or plastic strain $\left(\varepsilon_{\text {craze stablity }}\right)$ for $30 \mathrm{~nm}$ thick PS films by subtracting the strain at where maximum stress $\left(\varepsilon_{\text {yield }}\right)$ occurs from the maximum strain $\left(\varepsilon_{\text {yield }}\right)$ (Equation 1).

$$
\varepsilon_{\text {craze stablity }}=\varepsilon_{\max }-\varepsilon_{\text {yield }}
$$

\section{Comparison of TUTTUT and TUFF sample preparation and sample loading}

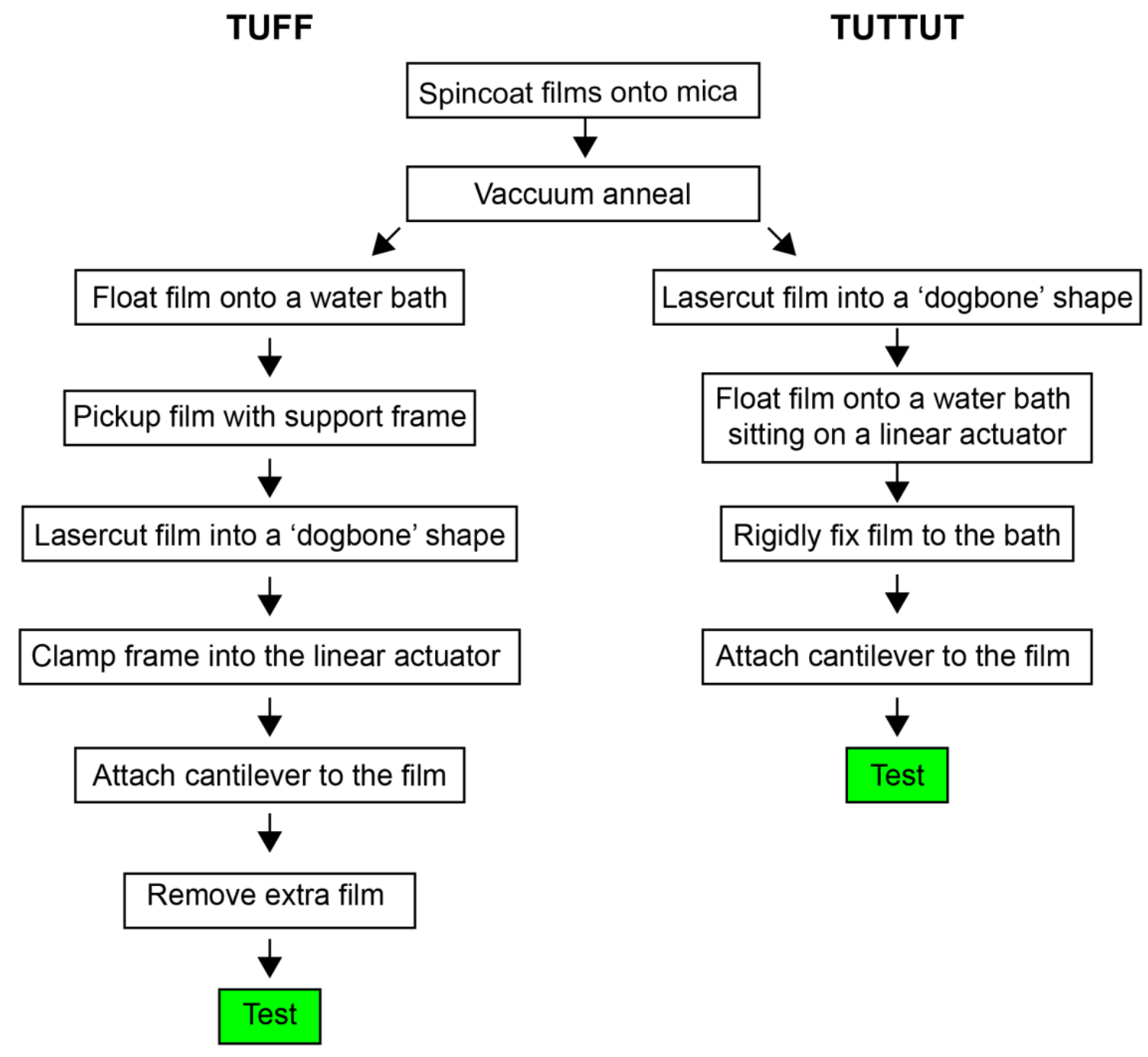

Figure S2. Flowchart comparison of the sample preparation and loading conditions between TUFF and TUTTUT. 


\section{Supplemental Movie Captions}

Movie S1: Demonstration of floating polystyrene film, picking up film (Film is labelled TUFF with permanent marker for visualization purposes), laser cutting the film, and loading into TUFF

Movie S2: Crazing formation and corresponding stress-strain response of $104 \mathrm{~nm}$ thick 151.5

$\mathrm{kDa}$ freestanding polystyrene film

Movie S3: Crazing formation and corresponding stress-strain response of $30 \mathrm{~nm}$ thick $151.5 \mathrm{kDa}$ freestanding polystyrene film

Movie S4: Crazing and tear formation, and corresponding stress-strain response of $30 \mathrm{~nm}$ thick $1007 \mathrm{kDa}$ freestanding polystyrene film

\section{References:}

(1) Bay, R. K.; Shimomura, S.; Liu, Y.; Ilton, M.; Crosby, A. J. Confinement Effect on Strain Localizations in Glassy Polymer Films. Macromolecules 2018, 51 (10), 3647-3653. 